\title{
An Assessment of Time Dependence of Defibrillator Benefit After Coronary Revascularization
}

\author{
Andreas C. Mauer, Daniel P. Morin, Eran S. Zacks, Matthew Janik, Shaun Ageno, \\ Steven M. Markowitz, Sei Iwai, Bruce B. Lerman and Kenneth M. Stein*
}

\author{
Maurice \& Corinne Greenberg Division of Cardiology, Department of Medicine, Weill Cornell Medical College, New \\ York, NY, USA
}

\begin{abstract}
Background: Previous studies have suggested that the benefit of implantable cardioverter defibrillator (ICD) implantation in patients following coronary revascularization $(\mathrm{CR})$ may be related to time elapsed since revascularization, with those receiving the device $>6$ months after the procedure deriving the greatest benefit.

Methods: We evaluated 163 patients (141 M/22 F, age $67 \pm 11 \mathrm{y}$, LVEF $30 \pm 8 \%)$ with a history of CR who underwent electrophysiology study (EPS) for risk stratification. ICD implantation was at the discretion of the treating physician. Occurrence of arrhythmia in ICD patients was ascertained from regular device clinic follow-up. Vital status was assessed using the National Death Index.

Results: 101 patients $(62 \%)$ had recent CR ( $\leq 6$ months before EPS) vs $62(38 \%)$ with remote CR (> 6 months). Median follow-up was $29 \pm 17$ months. There was no difference in arrhythmia-free survival $(\mathrm{p}=0.89,84$ [83\%] vs 52 [84\%] at 12 months), time to appropriate ICD therapy ( $\mathrm{p}=0.35,94$ [93\%] vs 55 [89\%] at 12 months), or overall survival ( $\mathrm{p}=0.15,91$ [90\%] vs 59 [95\%] at 12 months) between recent and remote CR patients.

Conclusions: Overall survival, arrhythmia-free survival, and time to first appropriate ICD therapy are similar between patients with recent and remote CR undergoing an EPS-guided approach to risk stratification.
\end{abstract}

Keywords: Defibrillation, ICD, electrophysiology, clinical.

\section{INTRODUCTION}

Sudden cardiac death (SCD) is a leading cause of death among patients with impaired left ventricular ejection fraction (LVEF) and coronary artery disease (CAD) [1]. Though coronary revascularization (CR) has contributed to improved prognosis in CAD, mortality attributable to SCD remains substantial in this population $[1,2]$.

Clinical trials of implantable cardioverter defibrillators (ICDs) have proven their benefit in patients with ischemic left ventricular dysfunction [3-5]. However, the Coronary Artery Bypass Graft Patch (CABG-Patch) trial showed no benefit to prophylactic ICD implantation at the time of coronary artery bypass graft (CABG) surgery [6], raising the question of optimal timing for ICD implantation relative to $\mathrm{CR}$. While major trials of ICD implantation have provided unequivocal evidence of the benefit of ICDs, timeframes for device implantation have varied. For instance, patients in the Multicenter UnSustained Tachycardia Trial (MUSTT) were eligible for study enrollment 4 days after the most recent myocardial infarction (MI) or CR [3], while those in the Multicenter Automatic Defibrillator Implantation Trial-I (MADIT-I) underwent implantation more than 3 weeks after MI, 2 months after CABG, and/or 3 months after percutaneous coronary intervention (PCI) [4], and patients in the Multicenter Automatic Defibrillator

*Address correspondence to this author at the Division of Cardiology, Weill Cornell Medical College, 520 East 70th Street, Starr-4, New York, NY 10021, USA; Tel: 212-746-2156; Fax: 212-746-6951;

E-mail: kstein@med.cornell.edu
Implantation Trial-II (MADIT-II) were excluded if they had a history of MI within 1 month or CR within 3 months of randomization [5].

Only one previous study has directly addressed the critical question of optimal timing for ICD implantation after revascularization. Goldenberg, et al. reported no benefit to ICD implantation in patients receiving ICDs within six months of the most recent revascularization, and suggested that the benefit of ICD implantation in this population was time-dependent [7]. In the present study, we compared the outcomes of ICD implantation after recent versus remote CR in a population with CAD, non-sustained ventricular tachycardia (NSVT), and LVEF $\leq 40 \%$ undergoing electrophysiology study (EPS) for risk stratification prior to the routine adoption of MADIT-II screening criteria [8].

\section{MATERIALS AND METHODOLOGY}

\section{Patients}

The patients in this study constituted a subgroup of a database that includes all patients who underwent EPS for risk stratification at the Cornell University Medical Center between December 1999 and January 2005. Inclusion criteria included $\mathrm{CAD}$, a history of $\mathrm{CR}, \mathrm{LVEF} \leq 40 \%$, documented NSVT, and sinus rhythm at the time of testing. CAD was defined by cardiac catheterization (at least one epicardial coronary artery with > $70 \%$ stenosis). LVEF was determined by echocardiography, nuclear gated imaging, or left ventriculography. No patient had a history of sustained ventricular arrhythmia or cardiac arrest. Patients were considered enrolled on the day of EPS. 


\section{Electrophysiological Testing}

All patients underwent EPS with up to triple ventricular extrastimuli at two different drive train cycle lengths from two right ventricular sites, in the basal state and during the infusion of isoproterenol. Positive EPS was defined as the induction of sustained monomorphic ventricular tachycardia with up to triple ventricular extrastimuli, or the induction of sustained polymorphic ventricular tachycardia or ventricular fibrillation with up to double ventricular extrastimuli. Sustained arrhythmias persisted for at least 30 seconds or were associated with syncope and/or hemodynamic compromise requiring either antitachycardia pacing or electrical cardioversion. ICD implantation, including device selection and programming, was at the discretion of the treating physician.

\section{Data Collection and Follow-Up}

A physician evaluated patient records to collect dates of CR most recently preceding EPS and whether the intervention was PCI or CABG. Patients who had received more than one intervention, whether multiple PCI, multiple CABG, or a combination of both, were classified as having received multiple CR and grouped according to their most recent intervention. As previous data had shown a benefit for ICD implantation only after 6 months had elapsed from CR [7], patients were segregated into two groups based on whether the most recent intervention preceding EPS was recent $(\leq 6$ months) or remote (> 6 months).

Demographic data including age, LVEF, sex, and medication use were collected by chart review. A physician evaluated each patient's most recent pre-EPS electrocardiogram for QRS duration and the presence of ventricular conduction delay as defined by standard criteria.
All patients were followed through chart review and query of the National Death Index. For patients who received ICDs or permanent pacemakers, periodic device interrogations (approximately every 3-6 months) were used to survey for arrhythmic events. Device electrograms were interpreted by blinded observers, and episodes for which therapy was delivered were classified as ventricular tachycardia (VT), ventricular fibrillation (VF), or inappropriate. The study endpoints were the composite endpoint of arrhythmia-free survival, ventricular arrhythmia requiring ICD therapy (antitachycardia pacing or high-voltage shock), and overall survival.

\section{Statistical Analysis}

For pairwise comparisons, Fisher's exact test was used for categorical variables, and the $t$-test was used to compare continuous variables. Multivariate Cox regression was performed to assess whether baseline covariates (age, LVEF, sex, medication use, QRS duration, mode of CR, and multiple CR) influenced arrhythmia-free survival, time to appropriate ICD therapy, and overall survival. The WilcoxonGehan statistic was then applied to life table analyses of recent and remote CR groups to assess arrhythmia-free survival, time to appropriate ICD discharge, and overall survival. Statistical analyses were performed using SPSS Version 12.0 for Windows (SPSS Inc., Chicago, IL). For all purposes, a $\mathrm{p}$ value $<0.05$ was required to reject the null hypothesis.

\section{RESULTS}

Baseline characteristics are summarized in Table 1. 163 patient records meeting stated criteria were identified. Of these, the indication for EPS was NSVT with syncope in 13 (8\%) patients and NSVT alone in $150(92 \%)$ patients. Me-

Table 1. Baseline Characteristics by Time from CR

\begin{tabular}{|c|c|c|c|c|}
\hline Age, y & $66.7( \pm 11.2)$ & $67.3( \pm 10.9)$ & $65.7( \pm 11.9)$ & 0.39 \\
\hline Male sex & $141(87 \%)$ & $87(86 \%)$ & $54(87 \%)$ & 1.00 \\
\hline Beta blocker & $138(85 \%)$ & $89(88 \%)$ & $49(79 \%)$ & 0.13 \\
\hline Statin & $97(60 \%)$ & $50(50 \%)$ & $47(76 \%)$ & 0.001 \\
\hline \multicolumn{5}{|l|}{ Most Recent CR } \\
\hline CABG & $78(48 \%)$ & $46(46 \%)$ & $32(52 \%)$ & 0.51 \\
\hline PCI & $85(52 \%)$ & $55(54 \%)$ & $30(48 \%)$ & 0.52 \\
\hline Inducible MVT, no VF & $143(88 \%)$ & $87(86 \%)$ & $56(90 \%)$ & 0.68 \\
\hline Inducible VF, no MVT & $2(1 \%)$ & $2(2 \%)$ & $0(0 \%)$ & N/A \\
\hline Inducible MVT and PVT/VF & $2(1 \%)$ & $0(0 \%)$ & $2(3 \%)$ & N/A \\
\hline
\end{tabular}

ACE = Angiotensin Converting Enzyme Inhibitor, ARB = Angiotensin Receptor Blocker, CABG = Coronary Artery Bypass Graft, CR = Coronary Revascularization, EOS = Electrophysiology Study, ICD = Implantable Cardioverter-Defibrillator, LVEF = Left Ventricular Ejection Fraction, MVT = Monomorphic Ventricular Tachycardia, PCI = Percutaneous Coronary Intervention, PVT/VF = Polymorphic Ventricular Tachycardia/Ventricular Fibrillation (with up to double ventricular extrastimuli). 
dian follow-up was $29 \pm 17$ months. 101 (62\%) patients had undergone $\mathrm{CR}$ within 6 months and $62(38 \%)$ had undergone CR more than 6 months prior to enrollment. $46(46 \%)$ recent and $32(52 \%)$ remote CR patients had most recently undergone CABG ( $\mathrm{p}=0.51$, Fig. 1). The most recent $\mathrm{CR}$ was PCI in $55(54 \%)$ recent and $30(48 \%)$ remote $\mathrm{CR}$ patients $(\mathrm{p}=$ $0.52) .17(17 \%)$ recent and $13(21 \%)$ remote CR patients had undergone multiple $\mathrm{CR}$ procedures $(\mathrm{p}=0.54)$. There were no significant difference between recent and remote CR groups in terms of age, LVEF, sex, QRS duration, beta blocker, or ACE/ARB use (all p>0.05). $50(50 \%)$ recent and 47 (76\%) remote CR patients were taking a statin at the time of EPS ( $p$ $<.001)$.

147 patients $(90 \%$,) had a positive EPS (89 [89\%] recent vs 58 [94\%] remote, $\mathrm{p}=0.29] .143(87 \%)$ had sustained monomorphic VT (86 [86\%] vs 56 [90\%], p = 0.68), $2(1 \%)$ had polymorphic VT/VF with single or double ventricular extrastimuli, and $2(1 \%)$ had both monomorphic VT and polymorphic VT/VF. 16 patients (10\%) had ICDs implanted despite a negative EPS (12 [12\%] vs 4 [6\%], $\mathrm{p}=0.74)$; ICDs were implanted in these patients due to individual providers' assessment of risk, incorporating risk factors including low LVEF and overall prognosis. Of these, 9 patients (6\%) had no inducible monomorphic VT or VF, 6 (4\%) had no inducible monomorphic VT but did have inducible polymorphic VT or VF with triple extrastimuli, and $1(1 \%)$ had polymorphic VT after rapid ventricular pacing. The rate of EPS positivity did not differ between recent and remote CR patients (89 [88\%] vs 58 [94\%], $\mathrm{p}=0.29$ ).

Results of multivariate analysis are summarized in Table 2. Only LVEF ( $p=0.01)$ and the use of Angiotensin Converting Enzyme inhibitors/Angiotensin receptor blockers $(\mathrm{ACE} / \mathrm{ARBs})(\mathrm{p}=0.03)$ independently predicted overall survival, while the influence of time from $\mathrm{CR}$ (recent $v s$ remote) was not significant $(\mathrm{p}=0.26)$. Multivariate analysis revealed no significant influence of baseline covariates on arrhythmia-free survival or time to appropriate ICD therapy (all $p$ > $0.05)$.

Outcomes are summarized in Table 3 . Over this period, $62(38 \%)$ patients reached the combined endpoint of VT/VF or death (39 [39\%] recent vs 23 [37\%] remote CR patients, $\mathrm{p}$ $=0.89$, Fig. 2). $33(20 \%)$ patients received appropriate ICD therapy for VT or VF (19 [19\%] recent vs 14 [23\%] remote CR patients, $p=0.35$, Fig. 3). The median time to appropriate device therapy was $11.8 \pm 17$ months. $41(25 \%)$ of 163 patients died (28 [27\%] recent vs 13 [21\%] remote CR patients, $\mathrm{p}=0.15$, Fig. 4).

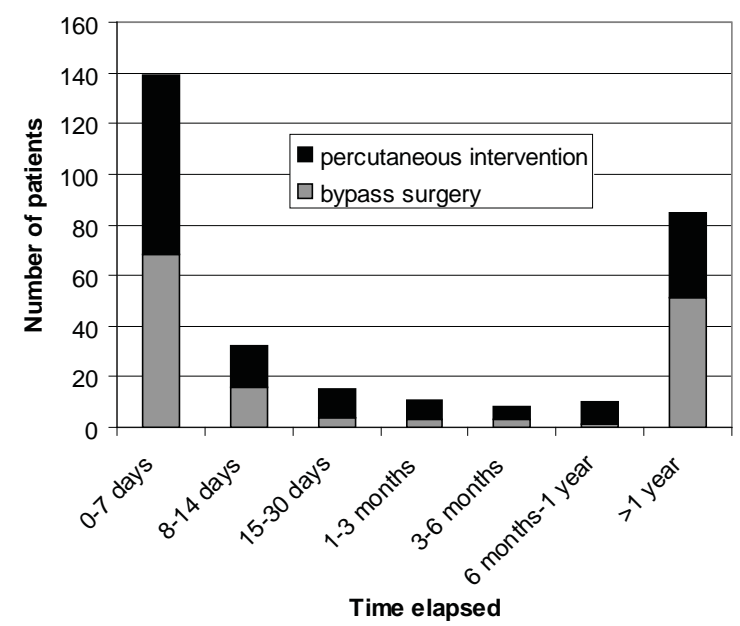

Fig. (1). Time to enrollment after coronary revascularization.

\section{DISCUSSION}

The principal finding of this study is that among patients with ischemic left ventricular dysfunction undergoing EPSguided risk stratification for prophylactic ICD implantation, arrhythmia-free and overall survival do not differ between patients with recent and remote CR.

The observation that patients in the present study had a similar prognosis after ICD implantation irrespective of the time since CR approaches the question of time dependence from a different perspective than a previous report demonstrating a mortality benefit of ICD implantation in the remote but not recent CR period [7]. This orthogonal viewpoint, reflected in differences in patient selection and study design, may contribute to the difference in findings. For instance, the previous study evaluated the MADIT-II population, for

Table 2. Multivariate Analysis

\begin{tabular}{|c|c|c|c|}
\hline Covariate* & Overall Survival ( $\boldsymbol{p})$ & Appropriate Device Therapy $(\boldsymbol{p})$ & Arrhythmia-Free Survival $(\boldsymbol{p})$ \\
\hline \hline CR (recent vs remote) & 0.26 & 0.73 & 0.72 \\
\hline LVEF & 0.01 & 0.46 & 0.45 \\
\hline ACE/ARB & 0.03 & 0.09 & 0.07 \\
\hline
\end{tabular}

*: $p$ for all other baseline covariates $>.05$

$\mathrm{ACE} / \mathrm{ARB}=$ Angiotensin converting enzyme inhibitor/Angiotensin receptor blocker; $\mathrm{CR}=$ Coronary revascularization; $\mathrm{LVEF}=\mathrm{Left}$ ventricular ejection fraction .

Table 3. Outcomes

\begin{tabular}{|c|c|c|c|c|}
\hline Outcome & Total $(\mathbf{n}=\mathbf{1 6 3})$ & Recent CR $(\leq \mathbf{6}$ Months $)(\mathbf{n}=\mathbf{1 0 1})$ & Remote CR $(>\mathbf{6}$ Months) $(\mathbf{n}=\mathbf{6 2})$ & $\boldsymbol{p}$ \\
\hline \hline VT, VF, or death & $62(38 \%)$ & $39(39 \%)$ & $23(37 \%)$ \\
\hline VT or VF & $33(20 \%)$ & $19(19 \%)$ & $14(23 \%)$ & 0.89 \\
\hline Death & $41(25 \%)$ & $28(27 \%)$ & $13(21 \%)$ & 0.35 \\
\hline
\end{tabular}

$\mathrm{CR}=$ Coronary Revascularization, ICD = Implantable Cardioverter-Defibrillator, VF = Ventricular Fibrillation, VT = Ventricular Tachycardia. 
whom NSVT and EPS were not mandatory and none of whom had been enrolled within 3 months of CR; in that study the only $14 \%$ of patients had an ICD implanted within 6 months of CR, as opposed to $62 \%$ in the present study.

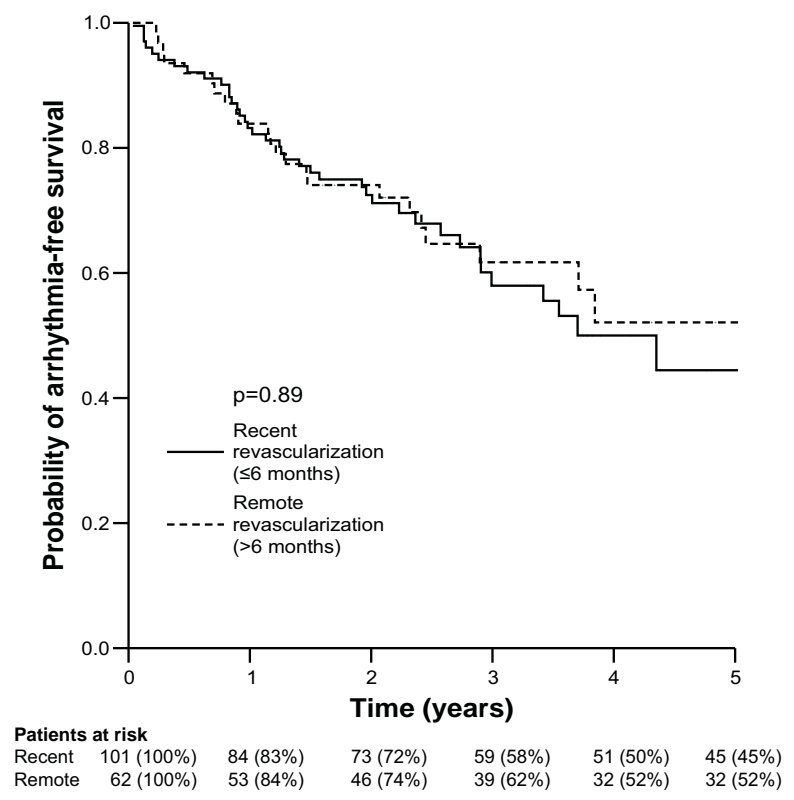

Fig. (2). Arrhvthmia-free survival.

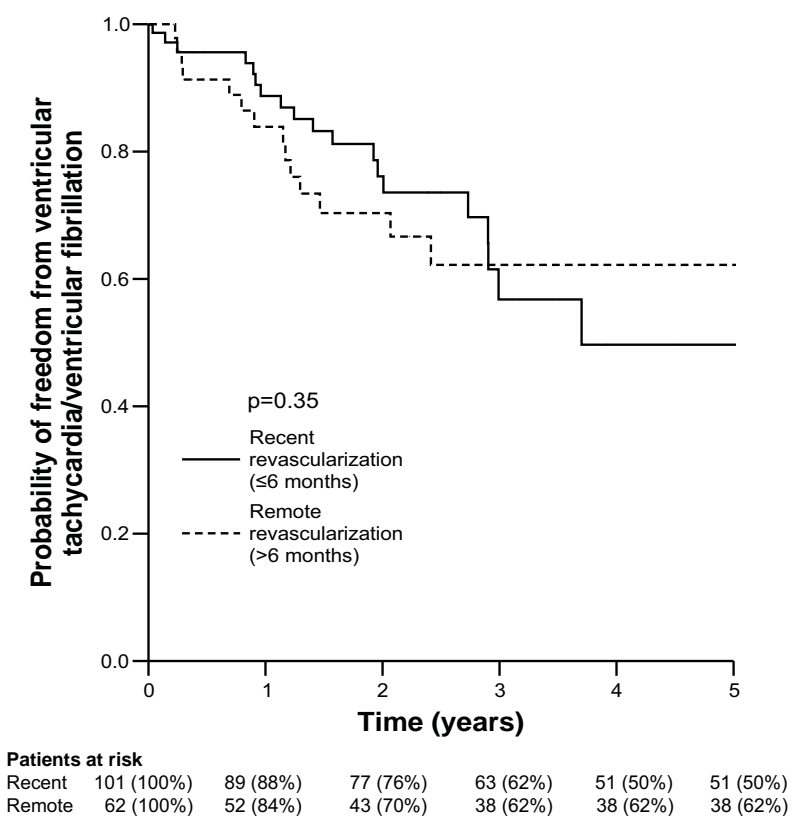

Fig. (3). Time to appropriate device therapy.

The EPS-guided risk stratification protocol employed here distinguishes the patients studied in this investigation from the population currently considered to be suitable for ICD implantation. Though guidelines for prophylactic ICD implantation stipulate that ICDs should be implanted no earlier than 3 months after the most recent revascularization [8], concordant with the results of CABG-Patch and Defibrillator in Acute Myocardial Infarction Trial (DINAMIT) [6, 9], current implantation guidelines do not stipulate an EPSguided approach to primary prophylaxis. Rather, the present study recalls the selection criteria of the MADIT-I/MUSTT era, in which EPS-guided therapy reduced SCD risk [3, 4]. The MUSTT registry demonstrated that patients with CAD, NSVT, and reduced LVEF who were inducible at EPS were at significantly higher risk for SCD than similar patients who were non-inducible [10] and that, although low LVEF and inducible tachyarrhythmia both predicted increased mortality risk, only inducibility predicted arrhythmic death [11]. Likewise, a study examining the prognostic significance of post-revascularization NSVT showed that patients who were inducible at EPS soon after revascularization had a high incidence of subsequent arrhythmic events [12]. Furthermore, though EPS was not mandatory in MADIT-II, studies of those patients who did undergo invasive testing demonstrated that inducibility was associated with increased likelihood for future VT [13].

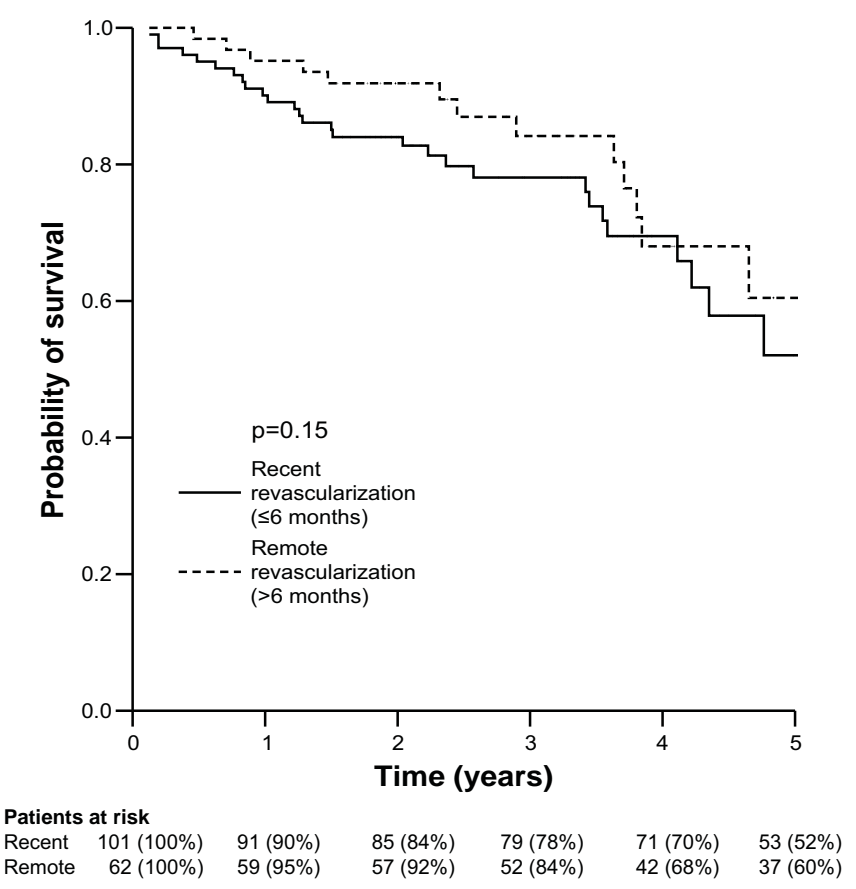

Fig. (4). Overall survival.

It is therefore plausible that the benefit of ICD implantation may be time dependent when patients are considered solely on the basis of ejection fraction, but that EPS-guided risk stratification and early ICD implantation may still be appropriate for patients with NSVT after CR. Support for this hypothesis can be found in one small study, the Betablocker Strategy plus ICD (BEST + ICD) trial, which demonstrated a trend in favor of early, EPS-guided ICD implantation in MI survivors [14]. Though these findings did not reach statistical significance, they suggested that EPS-guided risk stratification identified a small but high-risk group that benefited from ICD implantation within 1 month following myocardial infarction, a finding concordant with the results of the present study. Ongoing studies such as the Immediate Risk-Stratification Improves Survival (IRIS) trial [15] should provide clarity with respect to this important question. The 
multivariate analysis, which demonstrated that LVEF and ACE/ARB use (but not recent $v s$ remote CR) were significant predictors of survival is also noteworthy and underscores the need for careful medical management of patients with ischemic cardiomyopathy.

The present study is limited by several factors. Because ICD implantation was left to the treating physician at the time of testing, it is possible that different clinicians varied in their assessments for the need for ICD therapy, raising the possibility of unrecognized bias. Rapidly changing practice guidelines during the study's enrollment period (December 1999 to January 2005) may have contributed to this problem. Furthermore, as lipid-lowering therapy has been associated with reduced tachyarrhythmia rates in patients with CAD and ICDs [16-18] the lower rates of statin use in the recent CR cohort may have contributed to excess mortality in that group, thereby biasing the study in favor of similar survival between study groups. We also report a relatively high rate of inducibility at EPS, a finding that is likely related to the inclusion of NSVT among the enrollment criteria but which may not be representative of all patients eligible for ICD implantation.

Nonetheless, the contrast with the previous report of time-dependence highlights the fundamentally different nature of patients with post-revascularization NSVT and depressed LVEF, and suggests a continued role for EPS-guided risk stratification and early ICD implantation in this population. If confirmed, this finding offers a promising opportunity for improved treatment of these patients.

\section{CONCLUSION}

In a population with CAD, NSVT, and depressed LVEF undergoing an EPS-guided approach to risk stratification, arrhythmia-free survival, time to appropriate ICD therapy, and overall survival are similar between patients receiving an ICD less than 6 months after CR and those receiving an ICD greater than 6 months after CR.

\section{ACKNOWLEDGEMENTS}

This work was supported in part by grants from the National Institutes of Health (ROI HL-56139), the Rosenfeld Heart Foundation, the Michael Wolk Foundation, the Raymond and Beverly Sackler Foundation, and New York Cardiology Associates.

\section{CONFLICT OF INTEREST}

Dr. Stein has received honoraria and research support from Medtronic, Boston Scientific, and St. Jude Medical.

\section{ABBREVIATIONS}

$\begin{array}{ll}\mathrm{ACE} & =\text { Angiotensin-converting enzyme } \\ \mathrm{ARB} & =\text { Angiotensin receptor blockers } \\ \mathrm{CABG} & =\text { Coronary artery bypass graft } \\ \mathrm{CAD} & =\text { Coronary artery disease } \\ \mathrm{CR} & =\text { Coronary revascularization } \\ \mathrm{EPS} & =\text { Electrophysiology study }\end{array}$
ICD $=$ Implantable cardioverter-defibrillator
LVEF $=$ Left ventricular ejection fraction
MI $=$ Myocardial infarction
NSVT $=$ Non-sustained ventricular tachycardia
$\mathrm{PCI}=$ Percutaneous coronary intervention
$\mathrm{SCD}=$ Sudden cardiac death
$\mathrm{VT}=$ Ventricular tachycardia
$\mathrm{VF}=$ Ventricular fibrillation

\section{REFERENCES}

[1] Zheng ZJ, Croft JB, Giles WH, Mensah GA. Sudden cardiac death in the United States, 1989 to 1998. Circulation 2001; 104: 2158-63.

[2] Makikallio TH, Barthel P, Schneider R, et al. Frequency of sudden cardiac death among acute myocardial infarction survivors with optimized medical and revascularization therapy. Am J Cardiol 2006; 97: 480-4.

[3] Buxton AE, Lee KL, Fisher JD, Josephson ME, Prystowsky EN, Hafley GA. A randomized study of the prevention of sudden death in patients with coronary artery disease. N Engl J Med 1999; 341: 1882-90.

[4] Moss AJ, Hall WJ, Cannom DS, et al. Improved survival with an implanted defibrillator in patients with coronary disease at high risk for ventricular arrhythmia. N Engl J Med 1996; 335: 1933-40.

[5] Moss AJ, Zareba W, Hall WJ, et al. Prophylactic implantation of a defibrillator in patients with myocardial infarction and reduced ejection fraction. N Engl J Med 2002; 346: 877-83.

[6] Bigger JT. For the Coronary Artery Bypasss Graft (CABG) Patch Trial Investigators. Prophylactic use of implanted cardiac defibrillators in patients at high risk for ventricular arrhythmias after coronary-artery bypass graft surgery. N Engl J Med 1997; 337: 156975.

[7] Goldenberg I, Moss AJ, McNitt S, et al. Time dependence of defibrillator benefit after coronary revascularization in the multicenter automatic defibrillator implantation trial (MADIT)-II. J Am Coll Cardiol 2006; 47: 1811-7.

[8] Department of Health and Human Services Centers for Medicaid and Medicare Services. 2005. Implantable Automatic Defibrillators. http: //www.cms.hhs.gov/transmittals/downloads/R29NCD. pdf [accessed November $4^{\text {th }}, 2008$ ].

[9] Hohnloser SH, Kuck KH, Dorian P, et al. Prophylactic use of an implantable cardioverter-defibrillator after acute myocardial infarction. N Engl J Med 2004; 351: 2481-8.

[10] Buxton AE, Lee KL, DiCarlo L, et al. Electrophysiologic testing to identify patients with coronary artery disease who are at risk for sudden death. N Engl J Med 2000; 342: 1937-45.

[11] Buxton AE, Lee KL, Hafley GE, et al. Relation of ejection fraction and inducible ventricular tachycardia to mode of death in patients with coronary artery disease: an analysis of patients enrolled in the multicenter unsustained tachycardia trial. Circulation 2002; 106 : 2466-72.

[12] Mittal S, Lomnitz DJ, Mirchandani S, et al. Prognostic significance of nonsustained ventricular tachycardia after revascularization. J Cardiovasc Electrophysiol 2002; 13: 342-6.

[13] Daubert JP, Zareba W, Hall WJ, et al. Predictive value of ventricular arrhythmia inducibility for subsequent ventricular tachycardia or ventricular fibrillation in multicenter automatic defibrillator implantation trial (MADIT) II patients. J Am Coll Cardiol 2006; 47 : 98-107.

[14] Raviele A, Bongiorni MG, Brignole M, et al. Early EPS/ICD strategy in survivors of acute myocardial infarction with severe left ventricular dysfunction on optimal beta-blocker treatment - the Beta-blocker Strategy plus ICD trial. Europace 2005; 7: 327-37.

[15] Steinbeck G, Andresen D, Senges J, Hoffmann E, Seidl K, Brachmann J. Immediate Risk-Stratification Improves Survival (IRIS): study protocol. Europace 2004; 6: 392-9. 
[16] Mitchell LB, Powell JL, Gillis AM, Kehl V, Hallstrom AP. Are lipid-lowering drugs also antiarrhythmic drugs? An analysis of the antiarrhythmics versus implantable defibrillators (AVID) trial. J Am Coll Cardiol 2003;42: 81-7.

[17] De Sutter J, Tavernier R, De Buzere M, Jordaens L, De Backer G. Lipi lowering drugs and recurrences of life-threatening ventricular arrhythmias in high-risk patients. J Am Coll Cardiol 2000; 36(3): 766-72.

[18] Vyas AK, Guo H, Moss AJ, et al. Reduction in ventricular tachyarrhythmias with statins in the multicenter automatic defibrillator implantation trial (MADIT)-II. J Am Coll Cardiol 2006; 47: 769-73.

(C) Mauer et al.; Licensee Bentham Open.

This is an open access article licensed under the terms of the Creative Commons Attribution Non-Commercial License (http://creativecommons.org/licenses/by$\mathrm{nc} / 3.0 /$ ) which permits unrestricted, non-commercial use, distribution and reproduction in any medium, provided the work is properly cited. 